\title{
Butterfly-fauna of Gulmarg, Kashmir, J\&K State.
}

\author{
Aijaz Ahmad Qureshi ${ }^{1}$, Rayees Ahmad Dar ${ }^{2}$, Shaheen Iqbal Tahir ${ }^{3}$ and R. C. \\ Bhagat $^{4}$ \\ ${ }^{1,4}$ P.G. Department of Zoology, University of Kashmir, Srinagar, Kashmir. \\ ${ }^{3}$ SKIMS, Soura, Srinagar, $J \& K$ \\ ${ }^{3}$ Government Higher Secondary School, Boys, Baramulla, Kashmir \\ *Present address of Corresponding author: Islamic University of Science and Technology, Awantipora, Jammu \\ and Kashmir.Email; draijazphd@ gmail.com
}

\begin{abstract}
Field surveys conducted at Gulmarg, Kashmir during the years of 2006-08 revealed presence of 31 butterfly species distributed in 8 families and 27 genera. During the present preliminary field investigations documented for the first time the dominant family was found to be Nymphalidae (36\%) followed by Pieridae (23\%), Satyridae (19\%), Lycaenidae (10\%) whereas Danaidae, Hesperiidae, Libytheidae and Papilionidae were represented by 3\% each. The butterflies were active from April to November and highest distribution was in summer season. Diversity was calculated by Shannon-Weiner, Simpson and Margalaf's diversity indices and the values obtained by these indices indicated that the area is rich in butterfly diversity. However, human pressure due to tremendous flow of tourists was found a major threat to the environment of the area. 11 hostplants distributed in 8 families and 11 genera are being reported for the first time and highest number of butterflies visited the members of Asteraceae.
\end{abstract}

Key Words: Gulmarg, Kashmir Valley, butterflies, distribution, diversity indices, host plants.

\section{Introduction:}

Butterflies along with moths belong to the order Lepidoptera (Lepis - Scale, pteron - wing) and are the only insects with wings covered with scales. They are among the most interesting groups of insects [7] and have been referred to as flagships and honorary birds. Among the invertebrate animals, they are one of the best studied group [11] and are considered indicators of environmental quality. They help in pollination and have a close association with plants. They have been a source of inspiration to designers, fashioners, poets and writers.

Gulmarg is a mountainous area extending between $74^{\circ} 28^{\prime}$ to $74^{\circ} 31^{\prime}$ East and $34^{\circ} 03^{\prime}$ to $33^{\circ} 58^{\prime}$ North [9]. Also called "Meadow of Flowers" the area is a part of district Baramulla of the Kashmir Valley situated at an altitude of $2730 \mathrm{~m}$ (meters above sea level) covering an area of $180 \mathrm{sq} . \mathrm{km}$. Besides being a Wildlife Sanctuary [2] it is known for its unparallel beauty and is rated as one of the matchless tourist spots of the world. Having a beautiful highland golf course, it is famous for golf hikes and is the premier resort for winter sports in the country. Gandola Cable Car has added another charm to Gulmarg being of highest cable car in Asia and one of the highest lift-served ski resorts in the world [1].

The rich floral wealth which represent the effect of altitudinal, topographic, biotic and edaphic influences, and gives a unique identification to the area is represented by 491 species viz. dicotyledons ( 424 species), monocotyledons (61 species) and gymnosperms (6 species) distributed under 291 genera and 62 families. The vegetation of the area include Aconitium cashmeriana, Anemone obtusiloba, Gentiana carinata, Anapies cuneifolia, Taraxacum officinale, Planrango himalaica Populus ciliate, Ulmus wallichiana, Berberis pachyanta, Salix wallicana, Rubus purpureus, Caltha palustris, Primula rosea, Circuim falconeri, Rhododendron hypenanthum [9]. However as compared to floral wealth, we have comparatively little information on the faunal elements including butterfly fauna of Gulmarg and no survey or study seems to have been conducted on the butterflies of Gulmarg. Although Wynter-Blyth's book titled "The butterflies of India" was published in 1957 but the work was compiled before 1947. He gave distribution of 4 butterfly species distributed in 3 families and 4 genera from Gulmarg. Keeping in view the significance of the area the present preliminary attempt to explore the butterfly fauna was taken.

\section{Materials And Methods:}

Random field surveys were conducted during 2006-2008 in different months/seasons. The adult butterflies were collected by insect collecting net and killed by placing in a killing bottle containing vapours of ethyl acetate and after that relaxing and setting were carried out. For the identification of butterflies, works of $[4$, $6,12]$ were followed. For common names of butterflies $[10,12]$ were followed. The nomenclature of host-plants is as per $[2,9]$. The collected specimens of the butterflies have been deposited in the Department of Zoology, 
University of Kashmir and are also with the first authors. The diversity of butterflies was calculated by using Shannon-Weiner, Simpson's and Margalef's diversity indices as per [5, 8, 13].

\section{Results And Discussion:}

In the present field study 31 species of butterflies distributed in 27 genera and 8 families are reported (Table-1). The families include Danaidae, Hesperiidae, Libytheidae and Papilionidae (1 genus and 1 species each), Lycaenidae (3 genera, 3 species), Pieridae (5 genera, 7 species), Satyridae (5 genera, 6 species) and Nymphalidae (10 genera, 11 species). Nymphalidae was found to be most dominant representing (36\%) followed by Pieridae (23\%), Satyridae (19\%), Lycaenidae (10\%), Libytheidae (3\%), Hesperiidae (3\%), Papilionidae (3\%) and Danaidae (3\%) of the butterfly wealth (Fig 1). Except Hesperiidae which showed presence from June to October, all the families were mostly active from May to August (Table 2). The distribution of butterflies was highest in summer season (June-August) representing all the families/genera/species, followed by autumn (September-November) and spring (March-May), whereas there was no butterfly activity observed during winter season (December-February) (Table 3). The present study added 30 species of butterflies to the Wynter-Blyth's observations.

The frequently traceable species include, Aglais cashmirensis, Aricia agestis, Aulocera brahminus, A. padma, Colias electo fieldi, C. erate, Cynthia cardui, Lycaena phlaeas, Pelopidas mathias, Pieris brassicae and Pontia daplidice. Others like Argyreus hyperbius, Aporia leucodice, Callerebia mani, Childrena childreni, Danaus chryssipus, Gonepteryx rhamni, Hypolimnas misippus, Issoria. lathonia, Junonia iphita, J. orithya, Kaniska canace, Lampides boeticus, Libythea lepita, Maniola pulchella, Melanitis phedima, Neptis hylas, Paplio machaon, Pararga eversmanni cashmiensis, Pieris canidia, and Vanessa indica were not frequently observed.

A total of 24 adult host-plants distributed in 18 families and 24 genera in which 11 new records are reported for the first time (Table 4). Highest dominant host plant family was Asteraceae followed by Lamaiceae. 11 species namely Aglais cashmirensis, Aricia agestis, Colias electo fieldi, Gonepteryx rhamni, Hypolimnas misippus, Junonia orithya, Lampides boeticus, Lycaena phlaeas, Papilio machaon, Pieris brassicae and Pontia daplidice showed puddling behaviour. The species which were sighted at the $1^{\text {st }}$ phase of Gandola Cable Car include Aglais cashmiriensis, Colias electo fieldi. Cynthia cardui, Kaniska canace, Lycaena phlaeas, Pieris brassicae, Papilio machaon, Phalanta phalanta and Pontia dapldiice. During the months of June and July of 2007, the members of Aulocera brahminus and Colias electo fieldii were found in hundreds mostly at damp vegetation. Among the 4 species reported by Wynter-Blyth (1957), only one namely Narrow-Banded Satyr, Aulocera brahminus (Blanchard) (Satyridae) was reported during the present study. The other 3 species viz. Chequered Blue, Philotes vicrama Moore (Lycaenidae), Jerdon's Silverspot, Clossiana jerdoni (Lang) (Nymphalidae) and Mountain Argus, Callerebia shallada Marshall \& de Niceville (Satyridae) were not traceable. Further Wynter-Blyth called Aulocera braminus as rare but the present field observations showed that it is a common species of the area.

The calculated values of diversity indices used are Shanon-Weiner Index from 3.241 (2007) to 3.821 (2006), Simpson's Index from 0.843 (2007) to 0.897 (2006) and Margalef's Index from 3.954 (2007) to 4.116 (2008) (Table 5). All the values obtained from these indices showed that the whole area is rich in butterfly abundance. The area of golf course, both phases of Gandola Cable Car and high tourist pressure spots were having less butterfly diversity as compared to rest of the area, since these areas were having highest anthropogenic pressures. Other areas were having dense vegetation and thus supported more butterflies. Our results coincided with the results of Khan et al 2004.

\section{Conclusion:}

The present study being first effort in exploring the butterfly wealth of this world recognized tourist spot observed that the area possesses a unique and diverse butterfly fauna. The highest abundance was seen at areas with less human disturbances, less vehicular movement, dense vegetation etc. However, it cannot be assessed whether the butterfly wealth of the area is increasing or decreasing. Being a tourist hub, it was observed that the area is under tremendous anthropogenic pressure and with the increase in overall tourist flow, the area will be put under more pressure and stress which shall be having deleterious effects on the environment of the area in the times to come. Hence to address these external challenges, research and tourist activities in the area need to be taken in tandem and giving serious thought to the ecotourism development of the area. Since butterflies are regarded as indicator taxa, the butterfly fauna of the area needs to be continuously monitored so that any changes in the environment which may occur in future can be identified and appropriate measures can be taken to counter them. 


\section{Acknowledgements:}

The authors are highly thankful to Head, Department of Zoology, University of Kashmir for all his help and Mr. Akhtar Hussain Malik (Curator), Department of Botany, University of Kashmir for identifying the host-plants.

\section{References:}

[1] Anonymous, 2009. Indicators of Regional Development 2007-08. Directorate of Economics and Statistics, Planning and Development Department, Government of Jammu and Kashmir. pp109.

[2] Dar, G. A., Bhagat, R. C. and Khan, M. A. 2002. Biodiversity of the Kashmir Himalaya (Valley Book House, Srinagar, India) pp399.

[3] Evans, B. W. H. 1932. The identification of Indian butterflies (Diocesan Press, Madras, India). pp454

[4] Haribal, M. 1992. The Butterflies of Sikkim Himalaya and their Natural History (Sikkim Nature Conservation Foundation (SNCF), Gangtok, Sikkim.) pp217.

[5] Khan, M. R., Ali, K., Bashir, I., Malik, I. A and Mir, A. 2004. Biodiversity of butterflies from districts Poonch and Sudhnoti, Azad Kashmir. Asian J. Plant Sci. 3(5):556-560.

[6] Kunte, K. 2006. India- A Lifescape, Butterflies of Peninsular India (Universities Press (India) Private Ltd. Hyderabad, India) pp254.

[7] Pajni, H. R., Rose, H. S. and Walia, V. K.2006. Butterflies of North-West India. Part-1. Atma Ram \& Sons, Chandigarh, India. pp115.

[8] Simpson, E. H. 1949. Measurement of Diversity. Nature.163:688.

[9] Singh, G. and Kachroo, P. 1987. Forest flora of Srinagar and plants of neighbourhood (Periodical Expert Book Agency, Vivek Vihar, Delhi, India) pp278.

[10] Varshney, R. K. 1983. Index Rhopalocera indica part II. Common names of butterflies from India and neighbouring countries. Rec. Zool. Surv. India, Occasional Paper No. 47. pp49.

[11] Varshney, R. K. 1993. Index Rhopalocera Indica. Part III. Genera of Butterflies from India and neighbouring countries (Lepidoptera: (A) Papilionidae, Pieridae and Danaidae). Oriental Insects. 27:347-372.

[12] Wynter-Blyth, M. A. 1957. Butterflies of the Indian Region (The Bombay Natural History Society, Bombay, India) pp523.

[13] Zar. J. H. 2006. Biostatistical Analysis (Pearson Education, Inc. USA) pp663.

(Table- 1): Butterflies of Gulmarg, Kashmir.

\begin{tabular}{|c|c|c|c|c|}
\hline S.No. & Scientific Name & Common Name & Flight Period & Host plants \\
\hline \multicolumn{5}{|c|}{ Family I: Danaidae } \\
\hline 1 & $\begin{array}{l}\text { Danaus chrysippus } \\
\text { Linnaeus }\end{array}$ & Plain Tiger & $\begin{array}{l}\text { May to } \\
\text { August }\end{array}$ & $\begin{array}{l}\text { Lantana sp., } \\
\text { *Tagetus patula }\end{array}$ \\
\hline \multicolumn{5}{|c|}{ Family II: Hesperiidae } \\
\hline 2 & $\begin{array}{l}\text { Pelopidas mathias } \\
\text { (Fabricius) }\end{array}$ & $\begin{array}{l}\text { Small Branded } \\
\text { Swift }\end{array}$ & $\begin{array}{l}\text { June to } \\
\text { October }\end{array}$ & $\begin{array}{l}\text { *Digitalis purpurea, } \\
\text { Grasses, }\end{array}$ \\
\hline \multicolumn{5}{|c|}{ Family III: Libytheidae } \\
\hline 3 & $\begin{array}{l}\text { Libythea lepita } \\
\text { Moore }\end{array}$ & Common Beak & $\begin{array}{l}\text { May to } \\
\text { September }\end{array}$ & $\begin{array}{l}\text { Celtis australis, } \\
* \text { Rubus ulmifolius }\end{array}$ \\
\hline \multicolumn{5}{|c|}{ Family IV: Lycaenidae } \\
\hline 4 & $\begin{array}{l}\text { Aricia agestis } \\
\text { (Denis and } \\
\text { Schiffermuller) }\end{array}$ & $\begin{array}{l}\text { Orange-Bordered } \\
\text { Argus }\end{array}$ & $\begin{array}{l}\text { May to } \\
\text { October }\end{array}$ & *Mentha longifolia \\
\hline 5 & $\begin{array}{l}\text { Lampides boeticus } \\
\text { Linnaeus }\end{array}$ & Pea Blue & $\begin{array}{l}\text { May to } \\
\text { September, }\end{array}$ & Vigna sp. \\
\hline 6 & $\begin{array}{l}\text { Lycaena phlaeas } \\
\text { (Linnaeus) }\end{array}$ & $\begin{array}{l}\text { Common } \\
\text { Copper }\end{array}$ & $\begin{array}{l}\text { May to } \\
\text { September }\end{array}$ & $\begin{array}{l}\text { Rumex nepalensis, } \\
\text { *Tagetus patula, }\end{array}$ \\
\hline \multicolumn{5}{|c|}{ Family V: Nymphalidae } \\
\hline 7 & $\begin{array}{l}\text { Aglais cashmirensis } \\
\text { (Kollar) }\end{array}$ & $\begin{array}{l}\text { Indian } \\
\text { Tortoiseshell }\end{array}$ & $\begin{array}{l}\text { March to } \\
\text { November }\end{array}$ & $\begin{array}{l}\text { *Digitalis purpurea, } \\
\text { Tagetus patula, } \\
\text { Taraxacum } \\
\text { officinale, } \\
\wedge \text { Urtica diocia, }\end{array}$ \\
\hline 8 & $\begin{array}{l}\text { Argyreus hyperbius } \\
\text { (Johanssen) }\end{array}$ & $\begin{array}{l}\text { Indian } \\
\text { Fritillary }\end{array}$ & $\begin{array}{l}\text { May to } \\
\text { September }\end{array}$ & Viola tricolor \\
\hline 9 & $\begin{array}{l}\text { Childrena childreni } \\
\text { (Gray) }\end{array}$ & $\begin{array}{l}\text { Large } \\
\text { Silverstripe }\end{array}$ & $\begin{array}{l}\text { May to } \\
\text { October }\end{array}$ & $\begin{array}{l}\text { *Budlleja asiatica } \\
\text { *Mentha longifolia, } \\
\text { Viola tricolor }\end{array}$ \\
\hline 10 & $\begin{array}{l}\text { Cynthia cardui } \\
\text { (Linnaeus) }\end{array}$ & $\begin{array}{l}\text { Painted } \\
\text { Lady }\end{array}$ & $\begin{array}{l}\text { April to } \\
\text { November }\end{array}$ & $\begin{array}{l}\text { Artemesia vulgaris, } \\
\text { Blumea } \text { sp., } \\
\text { *Tagetus petula, } \\
\text { *Thymus serpyllum, } \\
\text { Urtica diocia }\end{array}$ \\
\hline
\end{tabular}


Butterfly-fauna of Gulmarg, Kashmir, J\&K State.

\begin{tabular}{|c|c|c|c|c|}
\hline 11 & $\begin{array}{l}\text { Hypolimnas misippus } \\
\text { (Linnaeus) }\end{array}$ & $\begin{array}{l}\text { Danaid } \\
\text { Eggfly }\end{array}$ & $\begin{array}{l}\text { May to } \\
\text { October }\end{array}$ & $\begin{array}{l}\text { Portulaca } \\
\text { grandiflora }\end{array}$ \\
\hline 12 & $\begin{array}{l}\text { Issoria lathonia } \\
\text { (Linnaeus) }\end{array}$ & $\begin{array}{l}\text { Queen of } \\
\text { Spain Fritillary }\end{array}$ & $\begin{array}{l}\text { May to } \\
\text { September }\end{array}$ & $\begin{array}{l}\text { Taraxacum } \\
\text { officinale, } \\
\text { Viola } \text { sp., }\end{array}$ \\
\hline 13 & $\begin{array}{l}\text { Junonia iphita } \\
\text { (Crammer) }\end{array}$ & $\begin{array}{l}\text { Chocolate } \\
\text { Pansy }\end{array}$ & $\begin{array}{l}\text { May to } \\
\text { September }\end{array}$ & *Thymus serpyllum \\
\hline 14 & $\begin{array}{l}\text { *Junonia orithya } \\
\text { (Linnaeus) }\end{array}$ & Blue Pansy & $\begin{array}{l}\text { May to } \\
\text { October }\end{array}$ & $\begin{array}{l}\text { Grasses, } \\
* \text { Mentha longifolia, } \\
* \text { Rubus ulmifolius, }\end{array}$ \\
\hline 15 & $\begin{array}{l}\text { Kaniska canace } \\
\text { (Linnaeus) }\end{array}$ & $\begin{array}{l}\text { Blue } \\
\text { Admirable }\end{array}$ & $\begin{array}{l}\text { May to } \\
\text { September }\end{array}$ & Grasses \\
\hline 16 & $\begin{array}{l}\text { Neptis hylas } \\
\text { (Linnaeus) }\end{array}$ & $\begin{array}{l}\text { Common } \\
\text { Sailor }\end{array}$ & $\begin{array}{l}\text { May to } \\
\text { September }\end{array}$ & $\begin{array}{l}\text { *Mentha longifolia, } \\
\text { Rubus ulmifolius, } \\
\text { *Thymus serphyllum }\end{array}$ \\
\hline 17 & $\begin{array}{l}\text { Vanessa indica } \\
\text { (Herbst) }\end{array}$ & $\begin{array}{l}\text { Indian Red } \\
\text { Admirable }\end{array}$ & $\begin{array}{l}\text { May to } \\
\text { September }\end{array}$ & $\begin{array}{l}\text { *Digitalis purpurea, } \\
\wedge \text { Urtica diocia }\end{array}$ \\
\hline \multicolumn{5}{|c|}{ Family VI: Papilionidae } \\
\hline 18 & $\begin{array}{l}\text { Papilio machaon } \\
\text { Menetries }\end{array}$ & $\begin{array}{l}\text { Common } \\
\text { Yellow Swallowtail }\end{array}$ & $\begin{array}{l}\text { May to } \\
\text { September }\end{array}$ & Taraxacum officinale \\
\hline \multicolumn{5}{|c|}{ Family VII: Pieridae } \\
\hline 19 & $\begin{array}{l}\text { Aporia leucodice } \\
\text { (Eversmann) }\end{array}$ & $\begin{array}{l}\text { Himalayan } \\
\text { Blackvein }\end{array}$ & $\begin{array}{l}\text { April to } \\
\text { October }\end{array}$ & $\begin{array}{l}\text { *Thymus serpyllum, } \\
\text { *Viola tricolor }\end{array}$ \\
\hline 20 & $\begin{array}{l}\text { Colias electo fieldi } \\
\text { Menetries }\end{array}$ & $\begin{array}{l}\text { Dark } \\
\text { Clouded Yellow }\end{array}$ & $\begin{array}{l}\text { April to } \\
\text { November }\end{array}$ & $\begin{array}{l}\text { *Digitalis purpurea, } \\
\text { *Medicago } \\
\text { polymopha, } \\
\text { *Ranunculus sp., } \\
\text { *Tagetus patula, } \\
\text { Taraxacum officinale }\end{array}$ \\
\hline 21 & Colias erate Esper & $\begin{array}{l}\text { Pale } \\
\text { Clouded Yellow }\end{array}$ & $\begin{array}{l}\text { April to } \\
\text { October }\end{array}$ & $\begin{array}{l}\text { *Medicago } \\
\text { polymopha, } \\
* \text { Tagetus patula, } \\
\text { Taraxacum } \\
\text { officinale, }\end{array}$ \\
\hline 22 & $\begin{array}{l}\text { Gonepteryx rhamni } \\
\text { (Linnaeus) }\end{array}$ & $\begin{array}{l}\text { Common } \\
\text { Brimstone }\end{array}$ & $\begin{array}{l}\text { May to } \\
\text { September }\end{array}$ & Rhamnus sp., \\
\hline 23 & $\begin{array}{l}\text { Pieris brassicae } \\
\text { (Linnaeus) }\end{array}$ & $\begin{array}{l}\text { Large } \\
\text { Cabbage White }\end{array}$ & $\begin{array}{l}\text { March to } \\
\text { November }\end{array}$ & $\begin{array}{l}\text { *Digitalis purpurea, } \\
* \text { Medicago } \\
\text { polymopha, } \\
\text { *Mentha longifolium, } \\
\text { *Rubus ulmifolius, } \\
\text { *Tagetus patula, } \\
\text { *Taraxacum } \\
\text { officinale, } \\
\text { *Thymus serpyllum }\end{array}$ \\
\hline 24 & $\begin{array}{l}\text { Pieris canidia } \\
\text { (Sparrman) }\end{array}$ & $\begin{array}{l}\text { Indian } \\
\text { Cabbage White }\end{array}$ & $\begin{array}{l}\text { May to } \\
\text { October }\end{array}$ & Sisymbrium sp. \\
\hline 25 & $\begin{array}{l}\text { Pontia daplidice } \\
\text { (Linnaeus) }\end{array}$ & Bath White & $\begin{array}{l}\text { May to } \\
\text { October }\end{array}$ & $\begin{array}{l}\text { Rubus ulmifolius, } \\
\text { *Tagetus petula, } \\
\text { *Taraxacum } \\
\text { officinale, } \\
\text { *Thymus serpyllum, }\end{array}$ \\
\hline \multicolumn{5}{|c|}{ Family VIII: Satyridae } \\
\hline 26 & $\begin{array}{l}\text { Aulocera brahminus } \\
\text { (Blanchard) }\end{array}$ & $\begin{array}{l}\text { Narrow- } \\
\text { Banded Satyr }\end{array}$ & $\begin{array}{l}\text { May to } \\
\text { September }\end{array}$ & $\begin{array}{l}\text { *Carduus edelbergi, } \\
\text { *Mentha longifolia }\end{array}$ \\
\hline 27 & $\begin{array}{l}\text { Aulocera padma } \\
\text { (Kollar) }\end{array}$ & $\begin{array}{l}\text { Great } \\
\text { Satyr }\end{array}$ & $\begin{array}{l}\text { May to } \\
\text { September }\end{array}$ & Carduus edelbergi \\
\hline 28 & Callerebia mani (De & Yellow & May to & Grasses, \\
\hline
\end{tabular}


Butterfly-fauna of Gulmarg, Kashmir, J\&K State.

\begin{tabular}{|l|l|l|l|l|}
\hline & Niceville) & Argus & September & $\begin{array}{l}\text { *Datisca cannabina } \\
\text { *Origanum vulgare }\end{array}$ \\
\hline 29 & $\begin{array}{l}\text { Maniola pulchella } \\
\text { (Felder) }\end{array}$ & $\begin{array}{l}\text { Tawny } \\
\text { Meadowbrown }\end{array}$ & $\begin{array}{l}\text { June to } \\
\text { September }\end{array}$ & *Tagetus patula \\
\hline 30 & $\begin{array}{l}\text { Melanitis phedima } \\
\text { (Stoll) }\end{array}$ & $\begin{array}{l}\text { Dark } \\
\text { Evening Brown }\end{array}$ & $\begin{array}{l}\text { June to } \\
\text { September }\end{array}$ & Oryza sativa \\
\hline 31 & $\begin{array}{l}\text { Pararge } \\
\text { everesmanni } \\
\text { cashmiensis } \\
\text { Eversmann }\end{array}$ & Yellow Wall & $\begin{array}{l}\text { May to } \\
\text { September }\end{array}$ & Grasses \\
\hline
\end{tabular}

Abbreviations used in the table. * New record; ^ Larval food plant; sp- species.

(Table 2): Number of families/genera/species reported in each month.

\begin{tabular}{|l|l|l|l|}
\hline Months & Families & Genera & Species \\
\hline January & 0 & 0 & 0 \\
\hline February & 0 & 0 & 0 \\
\hline March & 2 & 2 & 2 \\
\hline April & 2 & 4 & 5 \\
\hline May & 7 & 23 & 28 \\
\hline June & 8 & 27 & 31 \\
\hline July & 8 & 27 & 31 \\
\hline August & 8 & 27 & 31 \\
\hline September & 7 & 26 & 30 \\
\hline October & 5 & 12 & 13 \\
\hline November & 2 & 4 & 4 \\
\hline December & 0 & 0 & 0 \\
\hline
\end{tabular}

(Table 3): Number of families/genera/species in different seasons.

\begin{tabular}{|l|l|l|l|}
\hline Season & Family(ies) & Genus/Genera & Species \\
\hline Winter & 0 & 0 & 0 \\
\hline Spring & 7 & 23 & 28 \\
\hline Summer & 8 & 27 & 31 \\
\hline Autumn & 7 & 26 & 30 \\
\hline
\end{tabular}

(Table 4): Taxonomic list of host-plants.

\begin{tabular}{|l|l|l|}
\hline S.No. & Plant Family & Host-plant (s) \\
\hline 1 & Asteraceae & $\begin{array}{l}\text { Artemesia vulgaris, Blumea sp., Carduus edelbergi, Tagetus patula, } \\
\text { Taraxacum officinale }\end{array}$ \\
\hline 2 & Brassicaceae & Sisymbrium sp \\
\hline 3 & Buddlejaceae & Buddleja asiatica \\
\hline 4 & Datiscaceae & Datisca cannabina \\
\hline 5 & Celtaceae & Celtis australis \\
\hline 6 & Fabaceae & Medicago polymorpha, \\
\hline 7 & Lamiaceae & Mentha longifolia, Origanum vulgare Thymus serpyllum \\
\hline 8 & Leguminaceae & Vigna sinensis \\
\hline 9 & Plantaginaceae & Digitalis purpurea \\
\hline 10 & Poaceae & Oryza sativa \\
\hline 11 & Polygonaceae & Rumex nepalensis \\
\hline 12 & Portulacaceae & Portulacca oleracea \\
\hline 13 & Ranunculaceae & Ranunculus sp. \\
\hline 14 & Rhamnaceae & Rhamnus sp. \\
\hline 15 & Rosaceae & Rubus ulmifolius \\
\hline 16 & Urticaceae & Urtica diocia \\
\hline 17 & Verbenaceae & Lantana sp. \\
\hline 18 & Violaceae & Viola tricolor \\
\hline
\end{tabular}


(Table 5). Calculated values of diversity indices.

\begin{tabular}{|c|c|c|c|}
\hline Year & $\begin{array}{c}\text { Shannon- } \\
\text { Weiner } \\
\text { Index }\end{array}$ & $\begin{array}{c}\text { Simpson } \\
\text { Index }\end{array}$ & $\begin{array}{c}\text { Margalef's } \\
\text { Index }\end{array}$ \\
\hline $\mathbf{2 0 0 6}$ & 3.821 & 0.897 & 4.091 \\
\hline $\mathbf{2 0 0 7}$ & 3.241 & 0.843 & 3.954 \\
\hline $\mathbf{2 0 0 8}$ & 3.603 & 0.848 & 4.116 \\
\hline
\end{tabular}

Figure 1. Family wise abundance of butterflies at Gulmarg.

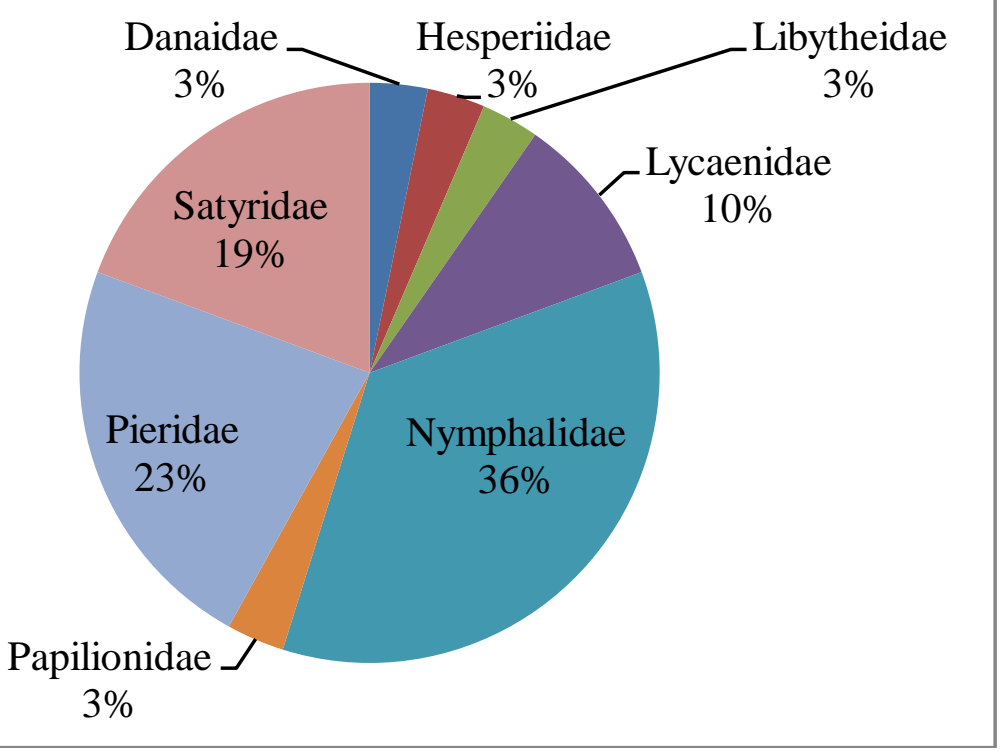

